\title{
Isolation and characterization of human skin-derived precursors
}

\author{
An Xie ${ }^{1}$, Yang Wang ${ }^{1}$, Li-Xing Zhang ${ }^{1}$, Yuan-Lei Lou ${ }^{1}$, Yu-Xia Liu ${ }^{1}$ \\ ${ }^{1}$ Institute of Urology, the First Affiliated Hospital of Nanchang University, Nanchang 330006, China
}

Human skin-derived precursors (SKPs), isolated and expanded from human skin, are known to be capable of differentiating into neural-like cells, which might provide an accessible and autologous source of neural crestderived cell types for the treatment of injured or diseased nervous system. SKPs are reported to be cultured in suspension as neurospheres. These neurosphere-like aggregates expand slowly and passage approximately every 2-3 weeks, which makes it hard to get enough cells for clinical practice in time. Therefore, the present study was focused on exploring a method for rapid expansion of precursors from the children dermis and observing their potential of differentiating into neural-like cells. Initially, cells were isolated and cultured in suspension as neurospheres in the presence of the mitogens including basic fibroblast growth factor (bFGF) and epidermal growth factor (EGF), and B27 is added instead of serum. After 4 passages, the cells were transferred to the serum-containing medium without mitogens to let the cells adhere to the bottom and grow as a monolayer. The population doubled about every 3 days and maintained for at least 30 passages. Reverse transcriptionpolymerase chain reaction (RT-PCR) results demonstrated that marker genes expressed in human SKPs cultured in suspension, such as nestin, vimentin, fibronectin, transcription factor genes slug, snail and p75 neurotrophin receptor (p75NTR) gene were still all expressed. To assess whether such cells still possess neural differentiation potential, retinoic acid (RA) was applied. In addition to the morphological changes, the results of immunofluorescence assays also showed that cells expressing neuron specific enolase (NSE) were found and some other cells expressing glial fibrillary acidic protein (GFAP), a character protein of schwann cells and astrocytes. In summary, we provide a method for rapid expansion of precursor cells with neural differentiation potential from human skin tissue. It has important implications for clinical practice that the precursor cells possess the ability to generate large numbers of neural precursors from a readily accessible, potentially autologous human source.

Keywords: skin-derived precursors, cell culture, neural-like cells

Cell Research (2008) 18:s110. doi: 10.1038/cr.2008.200; published online 4 August 2008

Correspondence: Yang Wang

E-mail: wangy63cn@sina.com 